\title{
Daftar-Gejii-Jafaris method for linear and nonlin- ear third order fractional differential equation
}

\author{
Mahmut Modanli \\ Department of Mathematics, Faculty of Art and Science, Harran University, 63200, Turkey.
}

\begin{abstract}
Numerical solution of the third order fractional differential equation is obtained by using DGJ (Daftardar-GejiiJafaris) method. Providing DGJ method converges, it is shown that obtained approximate solution is effective which is close to the exact solution or the exact solution. An example explained this method is presented. The proposed method is implemented for the approximation solution of the third order nonlinear fractional partial differential equations. An example which shows the method is unsuitable and inconsistent is given.
\end{abstract}

Keywords: DGJ method, third order fractional differential equation, nonlinear differential equation, convergence, numerical solution.

2010 MSC: 35R11, 65M99.

(C)2019 All rights reserved.

\section{Introduction}

Fractional partial differential equations have gained considerable importance recently in the literature. These equations have significant applications in finance, applied sciences, seismology engineering, physics and biology [1, 18, 19, 21, 23, 32]. Fractional differential equations can be solved separately depending on time and space variables. There are some methods for approximate solutions of fractional differential equations depending space and time variables $[4-6,25,26,33]$. Some of these methods are radial basis functions, Chebyshew Tau method, thin plate splines method, variational iteration method, finite difference schemes method, onedimensional sine-Gordon equation and DGJ method [2, 14, 22, 27, 29]. Also, one can find various good numerical solutions of the fractional differential equations in [7-10, 15-17, 24, 28, 34-40] etc.

Beside these facts, DGJ method is a good and effective mathod and has been attractive method recently. In $[11,12,30]$, this method was used for evolution and nonlinear functional equation

Email address: mmodanli@harran.edu.tr (Mahmut Modanli)

doi: $10.22436 / \mathrm{mns} .04 .01 .03$

Received: 2019-01-22 Revised: 2019-03-20 Accepted: 2019-04-05 
and fractional order linear, nonlinear systems. In [31], the telegraph equation was solved by DGJ method. Furthermore this method was applied to solve fractional heat-like and wave-like models with variable coefficients in [3].

In the present paper, we shall investigate the initial-boundary value problems of third-order fractional differential equations defined by Caputo derivative as follows:

$$
\left\{\begin{array}{l}
\frac{\partial^{3} u(t, x)}{\partial t^{3}}+\frac{\partial^{\alpha} u(t, x)}{\partial t^{\alpha}}+u(t, x)=\lambda \frac{\partial^{2} u(t, x)}{\partial x^{2}}+f(t, x), \\
0<x<L, 0<t<T, 0<\alpha \leqslant 1, \\
u(0, x)=g_{1}(x), u_{t}(0, x)=g_{2}(x), u_{t t}(0, x)=g_{3}(x), 0 \leqslant t \leqslant T, \\
u\left(t, X_{L}\right)=r_{1}(t), u\left(t, X_{R}\right)=r_{2}(t), X_{L}<x<X_{R} .
\end{array}\right.
$$

Here $\lambda$ is known constant coefficient, $g_{1}, g_{2}, g_{3}, r_{1}$ and $r_{2}$ are known functions and $u$ is an unknown function.

DGJ method has been employed successfully to solve a variety of problems and it is simple and easy for applying in fractional differential equation (1.1). It is economical in terms of computer power/memory and does not involve tedious calculations such as Adomian polynomials [20].

Differently from the problem investigated in [3], we show that DGJ method could be applied to a third-order and non-linear fractional partial differential equation. With the help of examples given in this study, advantages and disadvantages of DGJ method are discussed.

Now, we give some basic definitions and properties of fractional calculus theory for DGJ method.

Definition 1.1. The definition of gamma function is given the following form:

$$
\Gamma(z)=\int_{0}^{\mathrm{t}} \mathrm{e}^{-\mathrm{t}} \mathrm{t}^{z-1} \mathrm{dt}, \quad \forall z \in \mathrm{C} .
$$

Definition 1.2. The Caputo fractional derivative $D_{t}^{\alpha} u(t, x)$ of order $\alpha$ with respect to time is defined as:

$$
\begin{aligned}
\frac{\partial^{\alpha} u(t, x)}{\partial t^{\alpha}}= & D_{t}^{\alpha} u(t, x) \\
= & \frac{1}{\Gamma(n-\alpha)} \int_{0}^{t} \frac{1}{(t-p)^{\alpha-n+1}} \frac{\partial^{\alpha} u(p, x)}{\partial p^{\alpha}} d p, \\
& (n-1<\alpha<n),
\end{aligned}
$$

and for $\alpha=\mathrm{n} \in \mathrm{N}$ defined as:

$$
D_{t}^{\alpha} u(t, x)=\frac{\partial^{\alpha} u(t, x)}{\partial t^{\alpha}}=\frac{\partial^{n} u(t, x)}{\partial t^{n}} .
$$

Definition 1.3. By using gamma function and the formula (1.2), the following formula can be written

$$
D_{t}^{\alpha}\left(t^{\beta}\right)=\frac{\Gamma(\beta+1)}{\Gamma(\beta-\alpha+1)} t^{\beta-\alpha}
$$




\section{DGJ iteration method}

In this section, we shall present some basic facts dealing DGJ Iteration Method.

Using the method in [31], we can write the general form as:

$$
u(x)=N(u(x))+f(x)
$$

where $\mathrm{N}$ is an operator, $\mathrm{f}$ is a known function and $x=\left(x_{1}, x_{2}, \cdots, x_{n}\right)$. A solution of $u$ the equation in (2.1) is given the following series form

$$
u(x)=\sum_{j=0}^{\infty} u_{j}(x)
$$

Therefore, the operator $\mathrm{N}$ can be written as

$$
\mathrm{N}\left(\sum_{j=0}^{\infty} u_{j}(x)\right)=\mathrm{N}\left(\mathrm{u}_{0}\right)+\sum_{j=1}^{\infty}\left\{\left(\sum_{i=0}^{j} u_{i}\right)-\mathrm{N}\left(\sum_{i=0}^{j-1} u_{i}\right)\right\}
$$

Putting (2.2) and (2.3) in (2.1), we have

$$
\sum_{j=0}^{\infty} u_{j}=f+N\left(u_{0}\right)+\sum_{j=1}^{\infty}\left\{N\left(\sum_{i=0}^{j} u_{j}\right)-N\left(\sum_{i=0}^{j-1} u_{j}\right)\right\}
$$

From the above formulas, the recurrence relation can be obtained as

$$
\left\{\begin{array}{l}
u_{0}=f \\
u_{1}=N\left(u_{0}\right) \\
u_{n+1}=N\left(u_{0}+u_{1}+\cdots+u_{n}\right)-N\left(u_{0}+u_{1}+\cdots+u_{n-1}\right), n=1,2, \ldots
\end{array}\right.
$$

Thus we see that

$$
\left(u_{1}+\cdots+u_{n+1}\right)=N\left(u_{0}+u_{1}+\cdots+u_{n}\right), n=1,2, \cdots,
$$

and

$$
\sum_{j=0}^{\infty} u_{j}=f+N\left(\sum_{j=0}^{\infty} u_{j}\right)
$$

The p-term approximation solution of the formulas (2.1) and (2.2) is given by $u=u_{0}+u_{1}+$ $\cdots+u_{p-1}$.

Now, we shall apply DGJ method to third order fractional differential equation. Hence, the initial value of this operator is $L_{t t t}=\frac{\partial^{3}}{\partial t^{3}}$. The inverse operator of $L_{t t t}$ becomes

$$
\mathrm{L}_{\mathrm{ttt}}^{-1}=\int_{0}^{\mathrm{t}} \int_{0}^{\mathrm{t}} \int_{0}^{\mathrm{t}}(.) \mathrm{dt} \mathrm{dtdt}
$$


Applying the inverse operator to both sides of (1.1), the following integral equation is obtained

$$
\begin{aligned}
u(t, x)= & u(t, 0)+\frac{\partial u(t, 0)}{\partial t}+\frac{\partial^{2} u(t, 0)}{\partial t^{2}}+\int_{0}^{t} \int_{0}^{t} \int_{0}^{t} f(t, x) d t d t d t \\
& +\int_{0}^{t} \int_{0}^{t} \int_{0}^{t}\left(\lambda \frac{\partial^{2} u(t, x)}{\partial x^{2}}-\frac{\partial^{\alpha} u(t, x)}{\partial t^{\alpha}}-u(t, x)\right) d t d t d t
\end{aligned}
$$

Using the initial conditions of (1.1) and (2.4), we get

$$
\begin{aligned}
u_{0} & =g_{1}+g_{2} t+g_{3} \frac{t^{2}}{2}+\int_{0}^{t} \int_{0}^{t} \int_{0}^{t} f(t, x) d t d t d t \\
u_{1} & =N\left(u_{0}\right)=\int_{0}^{t} \int_{0}^{t} \int_{0}^{t}\left(\lambda \frac{\partial^{2} u_{0}(t, x)}{\partial x^{2}}-\frac{\partial^{\alpha} u_{0}(t, x)}{\partial t^{\alpha}}-u_{0}(t, x)\right) d t d t d t, \\
u_{m+1} & =N\left(u_{0}+u_{1}+\cdots+u_{m}\right)-N\left(u_{0}+u_{1}+\cdots+u_{m-1}\right), m=1,2, \cdots
\end{aligned}
$$

For convergences of this method and convergeness conditions, we refer to [12].

\section{Numerical implementation}

In this section, the proposed method is implemented to solve some examples of variable form of the third order fractional partial differential equation. In the first example, obtained solution is equivalent to the exact solutions. In the Example 3.2, the method has been observed for some values are moving away from the exact solution while for some values approaching the exact solution.

Example 3.1. Investigate the following third order fractional partial differential equation for initial boundary value problems

$$
\left\{\begin{array}{l}
\frac{\partial^{3} u(t, x)}{\partial t^{3}}+\frac{\partial^{\frac{1}{2}} u(t, x)}{\partial t^{\frac{1}{2}}}+u(t, x)=\frac{\partial^{2} u(t, x)}{\partial x^{2}}+e^{x}\left(6+6 \frac{t^{\frac{5}{2}}}{\Gamma\left(\frac{7}{2}\right)}\right), \\
0<x, 0<t<1,0<\alpha \leqslant 1 \\
u(0, x)=u_{t}(0, x)=u_{t t}(0, x)=0,0 \leqslant t .
\end{array}\right.
$$

Using the formula (2.5) and initial condition of the formula (3.1) for DGJ method, we obtain

$$
\begin{aligned}
u_{0} & =g_{1}+g_{2} t+g_{3} \frac{t^{2}}{2}+\int_{0}^{t} \int_{0}^{t} \int_{0}^{t} e^{x}\left(6+6 \frac{s^{\frac{5}{2}}}{\Gamma\left(\frac{7}{2}\right)}\right) d s d s d s \\
& =6 e^{x} \int_{0}^{t} \int_{0}^{t}\left(s+\frac{s^{\frac{7}{2}}}{\Gamma\left(\frac{9}{2}\right)}\right) d s d s=6 e^{x} \int_{0}^{t}\left(\frac{s^{2}}{2}+\frac{s^{\frac{9}{2}}}{\Gamma\left(\frac{11}{2}\right)}\right) d s
\end{aligned}
$$




$$
\begin{aligned}
& =6 e^{x}\left(\frac{t^{3}}{6}+\frac{t^{\frac{11}{2}}}{\Gamma\left(\frac{13}{2}\right)}\right) \\
u_{1} & =N\left(u_{0}\right)=\iint_{0}^{t} \int_{0}^{t}\left(\frac{\partial^{2} u_{0}(s, x)}{\partial x^{2}}-\frac{\partial^{\alpha} u_{0}(s, x)}{\partial t^{\alpha}}-u_{0}(t, x)\right) d s d s d s \\
& =-6 e^{x} \int_{0}^{t} \int_{0}^{t} \int_{0}^{t}\left(\frac{s^{\frac{5}{2}}}{\Gamma\left(\frac{7}{2}\right)}+\frac{s^{5}}{\Gamma(6)}\right) d s d s d s \\
& =-6 e^{x}\left(\frac{t^{\frac{11}{2}}}{\Gamma\left(\frac{13}{2}\right)}+\frac{t^{8}}{\Gamma(9)}\right. \\
u_{2} & =6 e^{x}\left(\frac{t^{8}}{\Gamma(9)}+\frac{t^{\frac{21}{2}}}{\Gamma\left(\frac{23}{2}\right)}\right) \\
& \vdots \\
u_{n} & =(-1)^{n} 6 e^{x}\left(\frac{t^{3+5 n}}{\Gamma(4+5 n)}+\frac{t^{\frac{9}{2}}+5 n}{\Gamma\left(\frac{11}{2}+5 n\right)}\right)
\end{aligned}
$$

When $n \rightarrow \infty$, the result converges to exact solution as follow

$$
\begin{aligned}
u(t, x) & =\lim _{n \rightarrow \infty} \sum_{j=0}^{n} u_{j} \\
& =6 e^{x} \lim _{n \rightarrow \infty} \sum_{j=0}^{n}(-1)^{n} e^{x}\left(\frac{t^{3+5 n}}{\Gamma(4+5 n)}+\frac{t^{\frac{9}{2}+5 n}}{\Gamma\left(\frac{11}{2}+5 n\right)}\right) \\
& =6 e^{x}\left(\frac{t^{3}}{6}-\lim _{n \rightarrow \infty} \frac{t^{\frac{9}{2}+5 n}}{\Gamma\left(\frac{11}{2}+5 n\right)}\right) \\
& =e^{x} t^{3}, \\
& \text { for all } 0 \leqslant t \leqslant 1 .
\end{aligned}
$$

The solution $u(t, x)=e^{x} t^{3}$ obtained by DGJ method is the exact solution for the problem (3.1).

Example 3.2. Investigate the following nonlinear third order fractional partial differential equation for initial boundary value problems

$$
\left\{\begin{array}{l}
\frac{\partial^{3} u(t, x)}{\partial t^{3}}+\frac{\partial^{\frac{1}{2}} u(t, x)}{\partial t^{\frac{1}{2}}}=3 \frac{\partial^{2} u(t, x)}{\partial x^{2}} u(t, x)+6\left(x-x^{2}\right)\left(t^{6}+\frac{t^{\frac{5}{2}}}{\Gamma\left(\frac{7}{2}\right)}+1\right), \\
0<x, 0<t<1,0<\alpha \leqslant 1, \\
u(0, x)=u_{t}(0, x)=u_{t t}(0, x)=0,0 \leqslant t \leqslant 1, \\
u(t, 0)=u(t, 1)=0,0 \leqslant x \leqslant 1 .
\end{array}\right.
$$


Using the formula (2.5) and initial condition of the formula (3.1) for DGJ method, we have

$$
\begin{aligned}
u_{0} & =g_{1}+g_{2} t+g_{3} \frac{t^{2}}{2} \\
& +\int_{0}^{t} \int_{0}^{t} \int_{0}^{t} 6\left(x-x^{2}\right)\left(s^{6}+\frac{s^{\frac{5}{2}}}{\Gamma\left(\frac{7}{2}\right)}+1\right) d s d s d s \\
& =6\left(x-x^{2}\right) \int_{0}^{t} \int_{0}^{t}\left(\frac{s^{7}}{7}+\frac{s^{\frac{7}{2}}}{\Gamma\left(\frac{9}{2}\right)}+s\right) d s d s \\
& =6\left(x-x^{2}\right) \int_{0}^{t}\left(\frac{s^{8}}{7.8}+\frac{s^{\frac{9}{2}}}{\Gamma\left(\frac{11}{2}\right)}+\frac{s^{2}}{2}\right) d s \\
& =6\left(x-x^{2}\right)\left(\frac{t^{9}}{7.8 .9}+\frac{t^{\frac{11}{2}}}{\Gamma\left(\frac{13}{2}\right)}+\frac{t^{3}}{6}\right)
\end{aligned}
$$

and

$$
\begin{aligned}
u_{1}= & N\left(u_{0}\right) \\
= & \int_{0}^{t} \int_{0}^{t} \int_{0}^{t}\left(3 \frac{\partial^{2} u_{0}(t, x)}{\partial x^{2}} u(t, x)-\frac{\partial^{\alpha} u_{0}(t, x)}{\partial t^{\alpha}}\right) d s d s d s \\
= & -\left(x-x^{2}\right) \int_{0}^{t} \int_{0}^{t} \int_{0}^{t}\left(6 \frac{s^{\frac{29}{2}}}{7 \Gamma\left(\frac{13}{2}\right)}+\frac{s^{18}}{24.49}+\frac{s^{12}}{7}\right. \\
& +216 \frac{s^{11}}{\left(\Gamma\left(\frac{13}{2}\right)\right)^{2}}+\left(\frac{6 \cdot \Gamma(7)}{\Gamma(19 / 2)}+\frac{72}{\Gamma\left(\frac{13}{2}\right)}\right) s^{\frac{17}{2}} \\
& \left.+\frac{s^{\frac{11}{2}}}{\Gamma\left(\frac{13}{2}\right)}+\frac{s^{3}}{6}+6 t^{6}\right) d s d s d s .
\end{aligned}
$$

From that, we get

$$
\begin{aligned}
\mathrm{u}_{1}= & -\left(x-x^{2}\right)\left(\frac{48}{7.31 .33 .35 \cdot \Gamma\left(\frac{13}{2}\right)} \mathrm{t}^{\frac{35}{2}}+\frac{\mathrm{t}^{21}}{19.20 .21 .24 .49}+\frac{\mathrm{t}^{15}}{7.13 .14 .15}\right. \\
& +\frac{216}{12.13 .14 \cdot\left(\Gamma\left(\frac{13}{2}\right)\right)^{2}} \mathrm{t}^{14}+\left(\frac{6 . \Gamma(7)}{\Gamma(25 / 2)}+\frac{576}{19.21 .23 . \Gamma\left(\frac{13}{2}\right)}\right) \mathrm{t}^{\frac{23}{2}} \\
& \left.+\frac{\mathrm{t}^{\frac{17}{2}}}{\Gamma\left(\frac{19}{2}\right)}+\frac{\mathrm{t}^{6}}{480}+\frac{\mathrm{t}^{9}}{84}\right) .
\end{aligned}
$$

Using (3.3) and (3.5) formulas for the numerical results by DGJ method, we obtain the following error analysis Table 3 with

$$
\epsilon=\max \left|u_{\text {exact }}-\sum_{i=0}^{k} u_{i}\right|,
$$

for $i=0,1$. Here $u_{\text {exact }}(t, x)=\left(x-x^{2}\right) t^{3}$ is exact solution for the nonlinear problem (3.2) that can 
obtained by using Laplace transform method and $u_{i}$ are the approximation solutions by using DGJ method obtained the above procedure.

\begin{tabular}{|l|l|l|l|}
\hline$t, x$ & $u_{i}(k=1)$ & $u_{\text {exact }}$ & $\epsilon(k=1)$ \\
\hline$t=0.5, x=0.5$ & $-4.142853 \times 10^{3}$ & 0.0312500 & $4.142884 \times 10^{3}$ \\
\hline$t=0.25, x=0.25$ & 0.186714 & 0.002929 & 0.189644 \\
\hline$t=0.1, x=0.1$ & $8.976138 \times 10^{-5}$ & $9.000000 \times 10^{-5}$ & $2.386131 \times 10^{-7}$ \\
\hline$t=0.05, x=0.05$ & $5.937559 \times 10^{-6}$ & $5.937500 \times 10^{-6}$ & $5.975908 \times 10^{-11}$ \\
\hline$t=0.02, x=0.02$ & $1.568001 \times 10^{-7}$ & $1.568000 \times 10^{-7}$ & $1.822421 \times 10^{-13}$ \\
\hline$t=0.01, x=0.01$ & $9.900002 \times 10^{-9}$ & $9.900000 \times 10^{-9}$ & $2.042696 \times 10^{-15}$ \\
\hline$t=0.001, x=0.001$ & $9.990000 \times 10^{-13}$ & $9.990000 \times 10^{-13}$ & $6.563302 \times 10^{-22}$ \\
\hline
\end{tabular}

By helping the Matlab program, obtained numerical solution of this problem can be applied for further steps. Intervals becomes smaller, it is seen that the approximate solution is very close to each other with exact solution. On the other hand, obtained approximations results are far from the exact solution for $t=0.5$ and $x=0.5$ since proposed method is not convergence which is shown as below.

Following the methods in $[12,30]$, we obtain

$$
\begin{aligned}
& \| \mathrm{N}\left(\mathrm{u}_{0}(\mathrm{t}, x)\|=\|-\left(x-x^{2}\right) \int_{0}^{\mathrm{t}} \int_{0}^{\mathrm{t}} \int_{0}^{\mathrm{t}}\left(6 \frac{\mathrm{s}^{\frac{29}{2}}}{7 \Gamma\left(\frac{13}{2}\right)}+\frac{s^{18}}{24.49}+\frac{\mathrm{s}^{12}}{7}\right.\right. \\
& +216 \frac{s^{11}}{\left(\Gamma\left(\frac{13}{2}\right)\right)^{2}}+\left(\frac{6 \cdot \Gamma(7)}{\Gamma(19 / 2)}+\frac{72}{\Gamma\left(\frac{13}{2}\right)}\right) s^{\frac{17}{2}} \\
& \left.+\frac{s^{\frac{11}{2}}}{\Gamma\left(\frac{13}{2}\right)}+\frac{s^{3}}{6}+6 t^{6}\right) d s d s d s \| \\
& =\|\left(x-x^{2}\right) \frac{48}{7 \cdot 31 \cdot 33 \cdot 35 \cdot \Gamma\left(\frac{13}{2}\right)} t^{\frac{35}{2}}+\frac{t^{21}}{19 \cdot 20 \cdot 21 \cdot 24 \cdot 49} \\
& +\frac{\mathrm{t}^{15}}{7.13 .14 .15}+\frac{216}{12.13 \cdot 14 \cdot\left(\Gamma\left(\frac{13}{2}\right)\right)^{2}} \mathrm{t}^{14} \\
& +\left(\frac{6 . \Gamma(7)}{\Gamma(25 / 2)}+\frac{576}{19.21 \cdot 23 \cdot \Gamma\left(\frac{13}{2}\right)}\right) t^{\frac{23}{2}} \\
& \left.+\frac{t^{\frac{17}{2}}}{\Gamma\left(\frac{19}{2}\right)}+\frac{t^{6}}{480}+\frac{t^{9}}{84}\right) \\
& =0.0286<\frac{1}{e^{\prime}} \\
& \| N_{t}\left(u_{0}(t, x)\|=\|-\left(x-x^{2}\right)\left(\frac{24}{7 \cdot 31 \cdot 33 \cdot \Gamma\left(\frac{13}{2}\right)} t^{\frac{33}{2}}\right.\right. \\
& +\frac{\mathrm{t}^{20}}{19.20 .24 .49}+\frac{\mathrm{t}^{14}}{7.13 .14}+\frac{216}{12.13 \cdot\left(\Gamma\left(\frac{13}{2}\right)\right)^{2}} \mathrm{t}^{13}
\end{aligned}
$$




$$
\begin{aligned}
& +\left(\frac{6 . \Gamma(7)}{\Gamma(23 / 2)}+\frac{288}{19.21 . \Gamma\left(\frac{13}{2}\right)}\right) t^{\frac{21}{2}} \\
& \left.+\frac{t^{\frac{15}{2}}}{\Gamma\left(\frac{17}{2}\right)}+\frac{t^{5}}{80}+9 \frac{t^{8}}{84}\right) \| \\
= & 0.2410<\frac{1}{e} \\
& \vdots \\
N_{t t} & =2.386494384604877 \times 10^{10}>\frac{1}{e} .
\end{aligned}
$$

The last formula shows that Example 3.2 is not convergence for $n>1$ derivatives with respect to $t$ because of DGJ method is equivalent to Taylor series expansion around $u_{0}$ and Taylor series conditions are not satisfied as in reference [13].

The vast majority of articles so far have just written the advantages of numerical methods. In this study, both advantages and disadvantages of the method were examined. We see that in Example 3.2, the approximate solution is not convergence to the exact solution at $x=t=0.5$ (see Table 3). Also, figures deal with approximate and exact solutions are presented.

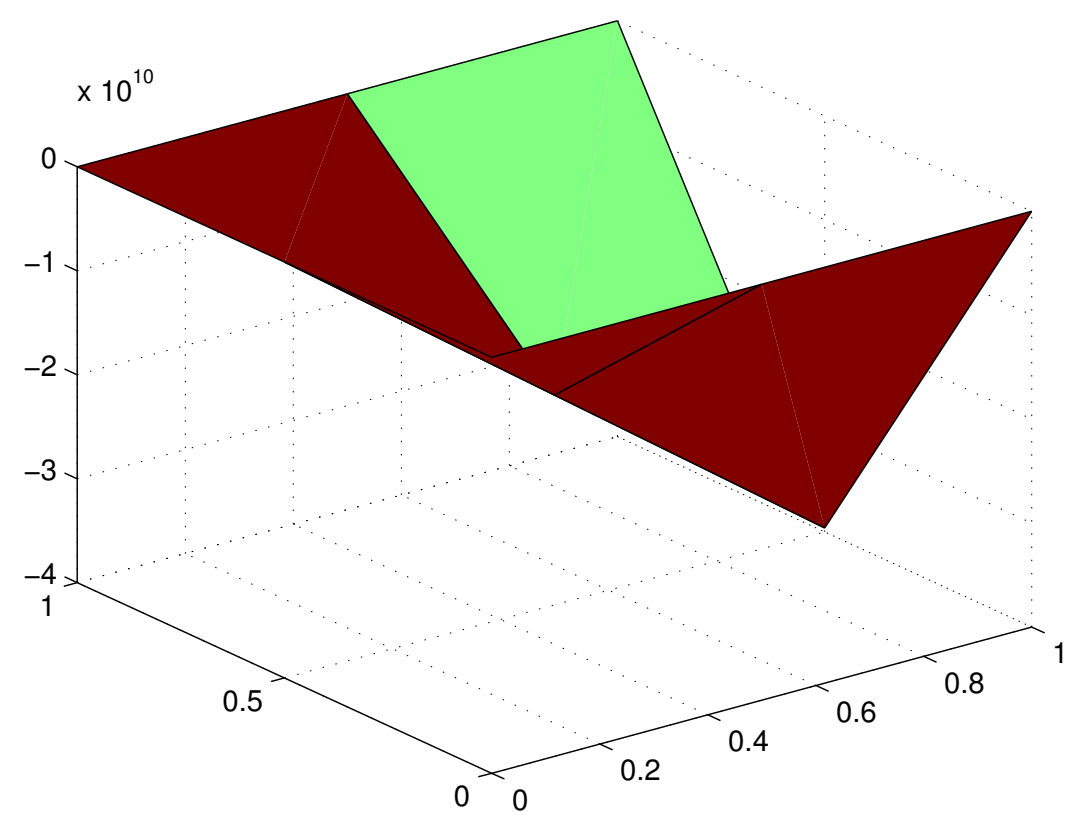

Figure 1: Figure of approximate solution

\section{Conclusion}

In this paper, the DGJ method is investigated for the third order linear and nonlinear fractional differential equation with Caputo fractional definition. This method gives the exact solution that is obtained by Laplace transform method depended initial-boundary value problems 


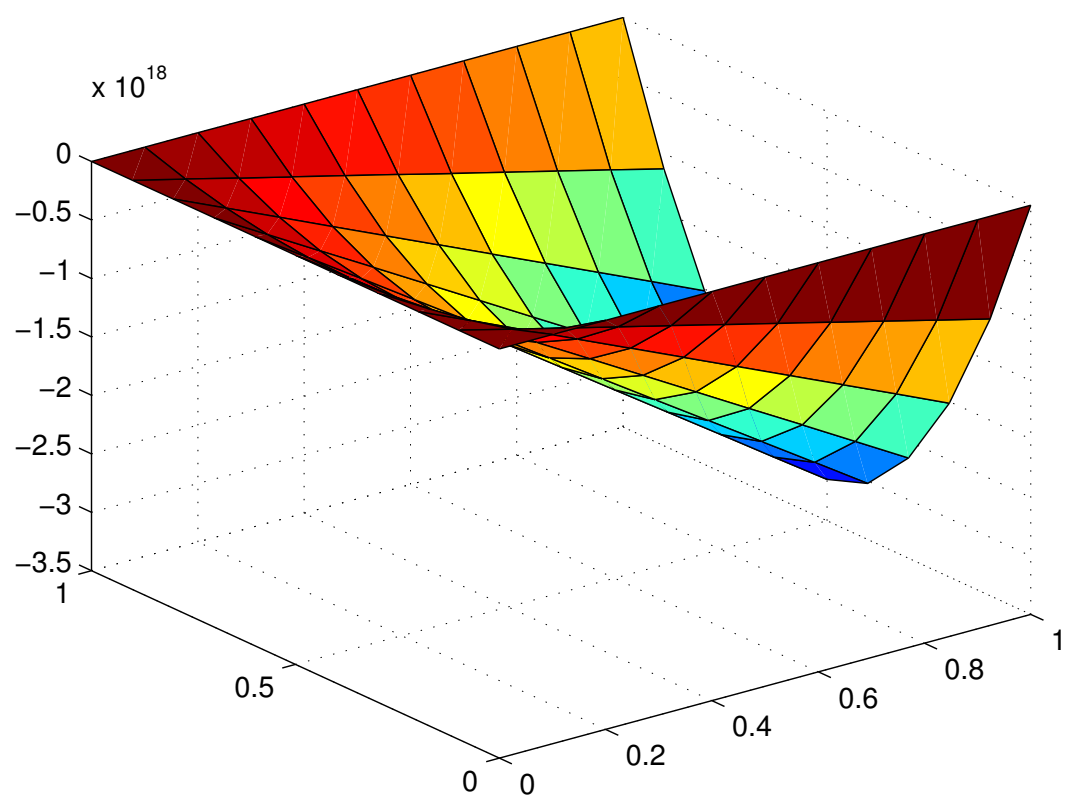

Figure 2: Figure of exact solution

for the first example. The second example is a nonlinear third order fractional partial differential equation which shows that this method gives close values to the exact solution at some intervals while blows up at some intervals. Approximate solutions for numerical experiments are found by this method. These results are compared with the exact solutions. MATLAB is used for numerical calculations for the Example 3.2.

\section{References}

[1] A. Akgül, D. Baleanu, F. Tchier, On the solutions of electrohydrodynamic flow with fractional differential equations by reproducing kernel method, Open Phys., 14 (2016), 685-689. 1

[2] A. Akgül, M. Inc, A. Kilicman, D. Baleanu, A new approach for one-dimensional sine-Gordon equation, Adv. Difference Equ., 2016 (2016), 20 pages. 1

[3] W. Al-Hayani, Daftardar-Jafari Method for Fractional Heat-Like and Wave-Like Equations with Variable Coefficients, Appl. Math., 8 (2017), 14 pages. 1, 1

[4] A. Ashyralyev, M. Modanli, A numerical solution for a telegraph equation, AIP Conference Proceedings, 2014 (2014), 300-304. 1

[5] A. Ashyralyev, M. Modanli, An operator method for telegraph partial differential and difference equations, Bound. Value Probl., 2015 (2015), 17 pages.

[6] A. Ashyralyev, M. Modanli, Nonlocal boundary value problem for telegraph equations, AIP Conference Proceedings, 2015 (2015), 4 pages. 1

[7] A. Atangana, D. Baleanu, New fractional derivatives with nonlocal and non-singular kernel: Theory and application to heat transfer model, Thermal Sci., 20 (2016), 763-769. 1

[8] H. M. Baskonus, H. Bulut, On the numerical solutions of some fractional ordinary differential equations by fractional Adams-Bashforth-Moulton method, Open Math., 13 (2015), 547-556.

[9] H. M. Baskonus, Z. Hammouch, T. Mekkaoui, H. Bulut, Chaos in the fractional order logistic delay system: Circuit realization and synchronization, AIP Conference Proceedings, 2016 (2016), 6 pages.

[10] H. M. Baskonus, G. Yel, H. Bulut, Novel wave surfaces to the fractional Zakharov-Kuznetsov-BenjaminBona-Mahony equation, AIP Conference Proceedings, 2017 (2017), 8 pages. 1 
[11] S. Bhalekar, Fractional Order Nonlinear Systems: Some Open Problems in Numerical Computations and Chaos Theory, Frontiers in fractional calculus, 2018 (2018), 342-347. 1

[12] S. Bhalekar, V. Daftardar-Gejji, Solving evolution equations using a new iterative method, Numer. Methods Partial Differential Equations, 26 (2010), 906-916. 1, 2, 3

[13] S. Bhalekar, V. Daftardar-Gejji, Convergence of the New Iterative Method, Int. J. Differ. Equ., 2011 (2011), 10 pages. 3

[14] J. Biazar, H. Ebrahimi, Z. Ayati, An approximation to the solution of telegraph equation by variational iteration method, Numer. Methods Partial Differential Equations, 25 (2009), 797-801. 1

[15] B. Boutarfa, A. Akgül, M. Inc, New approach for the Fornberg-Whitham type equations, J. Comput. Appl. Math., 312 (2017), 13-26. 1

[16] H. Bulut, T. A. Sulaiman, H. M. Baskonus, H. Rezazadeh, M. Eslami, M. Mirzazadeh, Optical solitons and other solutions to the conformable space-time fractional Fokas-Lenells equation, Optik, 172 (2018), 2027.

[17] H. Bulut, G. Yel, H. M. Başkonuş, An application of improved Bernoulli sub-equation function method to the nonlinear time-fractional burgers equation, Turkish J. Math. Comput. Sci., 5 (2016), 1-7. 1

[18] C. Çelik, M. Duman, Crank-Nicolson method for the fractional diffusion equation with the Riesz fractional derivative, J. Comput. Phys., 231 (2012), 1743-1750. 1

[19] I. I. Gorial, Numerical methods for fractional reaction-dispersion equation with Riesz space fractional derivative, Eng. Tech. J., 29 (2011), 709-715. 1

[20] M. Inc, M. T. Gencoglu, A. Akgül, Application of extended Adomian decomposition method and extended variational iteration method to Hirota-Satsuma coupled kdv equation, J. Adv. Phys., 6 (2017), 216-222. 1

[21] H. Jafari, V. Daftardar-Gejji, Solving linear and nonlinear fractional diffusion and wave equations by adomian decomposition, Appl. Math. Comput., 180 (2006), 488-497. 1

[22] M. Javidi, Chebyshev spectral collocation method for computing numerical solution of telegraph equation, Comput. Methods Differ. Equ., 1 (2013), 16-29. 1

[23] A. H. Khater, D. K. Callebaut, M. A. Helal, A. R. Seadawy, Variational method for the nonlinear dynamics of an elliptic magnetic stagnation line, Eur. Phys. J. D, 39 (2006), 237-245. 1

[24] Z. F. Kocak, H. Bulut, G. Yel, The solution of fractional wave equation by using modified trial equation method and homotopy analysis method, AIP Conference Proceedings, 2014 (2014), 504-512. 1

[25] M. Modanli, Two numerical methods for fractional partial differential equation with nonlocal boundary value problem, Adv. Difference Equ., 2018 (2018), 19 pages. 1

[26] M. Modanli, A. Akgül, Numerical solution of fractional telegraph differential equations by theta-method, Eur. Phys. J. Spec. Top., 226 (2017), 3693-3703. 1

[27] A. Mohebbi, A fourth-order finite difference scheme for the numerical solution of $1 D$ linear hyperbolic equation, Commun. Numer. Anal., 2013 (2013), 11 pages. 1

[28] N. Özdemir, M. Yavuz, Numerical solution of fractional Black-Scholes equation by using the multivariate Padé approximation, Acta Phys. Polonica A, 132 (2017), 1050-1053. 1

[29] A. Saadatmandi, M. Dehghan, Numerical solution of hyperbolic telegraph equation using the Chebyshev tau method, Numer. Methods Partial Differential Equations, 26 (2010), 239-252. 1

[30] M. Sari, A. Günay, G. Gürarslan, Approximate solutions of linear and non-linear diffusion equations by using Daftardar-Gejji-Jafari's method, Int. J. Math. Model. Numer. Optim., 2 (2011), 376-386. 1, 3

[31] M. Sari, A. Günay, G. Gürarslan, A solution to the telegraph equation by using DGJ method, Int. J. Nonlinear Sci., 17 (2014), 57-66. 1, 2

[32] A. Seadawy, A. Sayed, Soliton Solutions of Cubic-Quintic Nonlinear Schrodinger and Variant Boussinesq Equations by the First Integral Method, Filomat, 31 (2017), 4199-4208. 1

[33] L. Su, W. Wang, Z. Yang, Finite difference approximations for the fractional advection-diffusion equation, Phys. Lett. A., 373 (2009), 4405-4408. 1

[34] T. A. Sulaiman, H. Bulut, G. Yel, S. S. Atas, Optical solitons to the fractional perturbed RadhakrishnanKundu-Lakshmanan model, Opti. Quantum Electron., 50 (2018), 10 pages. 1

[35] T. A. Sulaiman, G. Yel, H. Bulut, M-fractional solitons and periodic wave solutions to the Hirota-Maccari system, Modern Phys. Lett. B, 33 (2019), 12 pages.

[36] P. Veeresha, D. G. Prakasha, H. M. Baskonus, New numerical surfaces to the mathematical model of cancer chemotherapy effect in Caputo fractional derivatives, Chaos, 29 (2019), 14 pages.

[37] M. Yavuz, N. Özdemir, A different approach to the European option pricing model with new fractional operator, Math. Model. Nat. Phenom., 13 (2018), 12 pages. 
[38] M. Yavuz, N. Özdemir, European vanilla option pricing model of fractional order without singular kernel, Frac. Fract., 2 (2018), 11 pages.

[39] M. Yavuz, N. Özdemir, Numerical inverse Laplace homotopy technique for fractional heat equations, Thermal Sci., 22 (2018), 185-194.

[40] M. Yavuz, N. Özdemir, H. M. Baskonus, Solutions of partial differential equations using the fractional operator involving Mittag-Leffler kernel, Eur. Phys. J. Plus, 133 (2018), 11 pages. 1 